\title{
Faculty and students perceive common tenets associated with medical student curriculum reform
}

\author{
Alexandria J. Bear1, Deborah Simpson 2, Diane Brown'3 , Dawn Bragg3, Karen Marcdante ${ }^{3}$ \\ ${ }^{1}$ Department of Internal Medicine, Medical College of Wisconsin, USA \\ ${ }^{2}$ Academic Administration, Aurora Health Care. Milwaukee, USA \\ ${ }^{3}$ Medical College of Wisconsin, Milwaukee, USA \\ Correspondence: Alexandria Bear, Internal Medicine Residency, Department of Medicine, Clinical Cancer Center at the Medical \\ College of Wisconsin, USA. Email: abear@mcw.edu
}

Accepted: August 31, 2013

\begin{abstract}
Objectives: Medical school faculty and students actively engaged in curriculum reform often experience angst. Change management literature emphasizes that grounding change in common values is critical to managing stakeholder angst and ultimately successful change. However the literature provides only limited descriptions of the shared underlying features as perceived by faculty and students associated with curriculum reform. This study sought to bridge this gap by identifying the underlying student and faculty beliefs associated with success in medical student education programs and reform.
\end{abstract}

Methods: A qualitative study approach using an appreciative inquiry interview methodology was selected given its proven success as an inquiry technique for change management. To identify cross-cutting curriculum success tenets, a purposeful sample of 24 stakeholders participating in an established curriculum and/or new integrated curriculum were selected: 12 students and 12 faculty seven of whom were curriculum/college leaders. Two rounds of appreciative inquiry interviews focusing on successes associated with medical student education were conducted. Interviews were digitally recorded, transcribed, coded, and then analyzed to delineate common themes and crosscutting tenets using constant comparative methodology.

Results: Analysis revealed six underlying themes associated with success for students and faculty: engagement of students and faculty in education, sense of community and collaboration, faculty/student relationships, active learning, faculty excitement/willingness to teach leads to impactful student learning, and identity/professional formation.

Conclusions: The identified tenets associated with successful medical student education programs can be used to manage a critical element of curriculum form: stakeholder change angst.

Keywords: Curriculum change planning, faculty development, educational evaluation, qualitative, appreciative inquiry

\section{Introduction}

The Flexner Report, published under the auspices of the Carnegie Foundation for the Advancement of Teaching, was the first to describe standards for medical education. ${ }^{1}$ Since the report's publication in 1910, American medical education has become increasingly standardized, consistent with Flexner's recommendation. ${ }^{2}$ Since 2000, more than 15 reports from diverse groups in the United States, Canada (e.g., American Medical Association, Association of American Medical Colleges, Institute of Medicine) ${ }^{3}$ and globally ${ }^{4}$ have emphasized that medical education and its trainees must be prepared to provide care that is more responsive to the needs of patients, populations, and shifting care delivery health care delivery strategies. In their reexamination of medical education 100 years post Flexner, Cooke, et al. emphasized that medical education must focus on standardization of learning outcomes with individualization of learning, integration of formal knowledge with clinical experience, development of the habits of inquiry and improvement, and addressing professional identity formation. ${ }^{5}$

The notable advancements in the science of learning ${ }^{5}$ and learning theory ${ }^{6}$ since Flexner's time have led to rec- 
ommendations including connecting formal knowledge with clinical experience through integration, addressing learner engagement, respecting learner differences, exploring professional roles, promoting opportunities for reflection, and establishing the learners' responsibility for their own learning. ${ }^{7}$ The combined changes in the health care environment (e.g., the triple aim of improving quality of care, decreasing costs and improving access) and the pedagogy of medical education support the need for continued change in medical school curriculum.

To address the goals of improving medicine, medical school curriculum change has become endemic globally. In the United States, 75\% of allopathic schools have initiated reform since 2005. Typically this change is towards an integrated curriculum with clinical experiences incorporated early in the first two years. ${ }^{1}$ Many educators, however, argue that more significant curriculum reform will be needed to prepare graduates to practice in health care settings that are responsive to the health needs of our communities and address the high costs and inefficiencies in our current health care model. ${ }^{8}$ Thus medical school curriculum will continue to change and those changes will continue to impact medical teachers and learners well into the future.

Indeed the changing environment in health care and medical education leads to predictable behaviors designed to resist change. Fear of the unknown, lack of information, fear of the loss of security or power, the difficulty in overcoming well established habits and the belief that there is no reason for change are often at the root of some of these behaviors. ${ }^{9}$ Despite these behaviors, successful change can be accomplished. While there are several models of how to address change, ${ }^{10,11}$ most emphasize the need to align the people involved around values. Stressing values of those involved can align the change with what those affected recognize as the importance of their work. Aligning values can help allay some of the fears and concerns by focusing on the foundational reason people perform the work.

Change requires individuals to shift from well-honed tasks (e.g., lecture) and expertise areas (e.g., discipline, specialty) to learn new roles, processes, and skills while managing their emotions. ${ }^{12,13}$ For example, Raja Bandaranayake reported that "the major problem with (changing to) integrated programmes is an attitudinal one, as teachers see integration as unjustifiably trespassing on territory that they consider their personal property." 14 This territoriality directly stems from a lack of control over changes. Students also experience curriculum change anxieties stemming from uncertainty about the depth, breadth, and expected mastery of the subject resulting in student focus on assessments to guide their study.

Apprehension during times of curriculum change has been further articulated as a progressive stage model, beginning with awareness of change and its personal impacts, change management, and ultimately refocusing on what individuals consider the benefits of the change while identifying new innovations that will continue to improve the curriculum. ${ }^{15}$ During the personal and management stages of change, individuals can be particularly resistant to change, often times focusing on what failed, rather than what worked. This "deficiency model" merely identifies the concern, and often does not delineate the root causes. ${ }^{14}$ For change to succeed, the initiative must be aligned with faculty and students beliefs and feelings about curriculum change $^{12}$ by reinforcing the shared tenets associated in medical education program success amongst students and faculty alike. The question then arises, are there shared success indicators /tenets that can help to mitigate faculty and student concerns during times of change?

A review of the literature yielded limited guidance on perceptions regarding shared success tenets associated with medical education programs during times of curriculum change, but did reveal a useful approach to identifying common success themes, appreciative inquiry.

Appreciative inquiry (AI) builds on learning what works well in an organization drawing from positive experiences to direct future endeavors. ${ }^{16}$ AI was originally described in the $1980 \mathrm{~s},{ }^{17,18}$ and has been subsequently characterized as both a personal and organizational change strategy which emerges from dialogue around what is working and then exploring those successes in detail. ${ }^{19}$ Over the last 30 years, AI, as a change management technique, has been successfully used to promote individual and organizational learning and change through its focus on when an organization is at its best in the past, present, and future. ${ }^{20} \mathrm{AI}$ complements the more conventional form of action research and is distinguished by its ability to incite generative learning. ${ }^{21}$

AI has been used in various medical educational and health professional spheres, focusing on professionalism, quality improvement, and interprofessional education. ${ }^{22,23}$ It has been credited with deepening students understanding of and appreciation for professionalism by eliciting common principles. ${ }^{19}$ AI has also been used to identify outstanding residents who exhibited exemplary sign-out practices, which subsequently drove the creation of new sign-out standards. ${ }^{22}$ Others report that AI changed perceptions of a medical education program and created "hopeful expectations" amongst the student body as it drove change. ${ }^{24}$

The literature focusing on the AI successes emerging during curriculum reform focuses on the development of a curriculum model, ${ }^{25-27}$ rather than successes during the change process. At the time of this study, the Medical College of Wisconsin (MCW) was in a unique position to examine cross-cutting success themes associated with our medical student educational program; the established curriculum (EC) for more than 170 first year students continued while a new integrated curriculum (NIC) enrolled 28 first year students. This distinctive study period provided an opportunity to focus on successes within each of the curricula from both student and faculty perspectives 
to yield perspectives on shared tenets underlying success within medical education.

The purpose of this study was to explicitly identify the underlying student and faculty beliefs associated with success in medical student education programs to illuminate shared tenets underlying curriculum change.

\section{Methods}

\section{Study design}

A qualitative study approach using an appreciative inquiry interview (AI) methodology was selected by a 14 -member evaluation work group given its proven success as an inquiry technique for change management. The work group was composed of 10 faculty representing the EC and NIC, residency program directors, faculty council committee members (e.g., academic standing, curriculum and evaluation committees), 3 medical educators, and 1 medical student.

\section{Participants and sample size}

The project was granted exempt status by the Medical College of Wisconsin Institutional Review Board. A purposeful sampling approach was used to assure that a breadth of perspectives were obtained in order to reach saturation consistent with our study aim, yet responsive to feasibility constraints common to qualitative data collection and analysis. ${ }^{28}$ The council identified key stakeholder groups reflecting a breadth of roles and perspectives regarding curriculum reform (Table 1). Faculty participants were then identified and selected from those groups based on their involvement as teachers and/or educational leaders to include a breadth of disciplines, specialties, teaching experience levels, and roles. All EC and NIC students were invited to participate. Student volunteer rates were high (EC=100/177; NIC=28/28); participant were selected to reflect the demographic and academic diversity of our class. Identified participants were contacted via e-mail by one of the authors and provided an overview of the process.

Those accepting the invitation to participate in two rounds of interviews during the 2010-11 academic year (Nov-Dec and April-June) were subsequently contacted by an assigned council member to be interviewed. No faculty member was assigned to students where they may be a perceived or actual conflict (e.g., academic standing committee, first year EC or NIC education director). The Medical College of Wisconsin's Institutional Review Board granted this project "exempt" status. This study qualified for exempt status due to the normal educational setting, voluntary nature of participation, and di-identification of protocol analysis (e.g., participant status as student or faculty and EC/NIC affiliation).

\section{Data collection procedures}

The AI interview protocol was created to parallel the standard appreciative inquiry protocol and included a detailed description of the AI process, recording consent, and description of how the information would be reported. ${ }^{18,19}$ The semi-structured interview began with a broad opening question for both faculty and students: "Step back and think of a recent experience related to our medical student education program. This experience should have occurred in the last 3-4 months. Pick a time when you have felt most engaged, alive, absorbed, excited, or proud." Follow-up probes asked the participant to describe the high point of the experience, who was involved, and what they did to specifically contribute to the success. A second section focused on what was occurring when our educational program is at its best, and then asked the interviewee to visualize what would be occurring in 2016 if our educational program was an amazing success. The interview protocol contained follow-up probes to explore the critical variables involved in the success (e.g., who was involved and what their specific contributions/roles were). To prepare interviewers, a mock interview was conducted at a council meeting, debriefed, and strategies for maintaining neutral and engaging interview interactions were highlighted.

Ten council members conducted 1-3 interviews during each round. Each participant consented to digital voice recording of the interview, which was then transcribed with line and page numbers along with a randomly assigned unique numeric identifier for tracking in lieu of name or other identifying information. Four authors independently analyzed 5-6 interview transcriptions using qualitative memos; three of the four had extensive previous experience in qualitative analysis (DS, DB, DB), and were participants in curriculum reform at MCW. The medical student (AB) was coached stepwise through the process, providing a student lens to coding and subsequent analysis. The 5th author (KM) reviewed results and provided clarity to themes and analysis.

Table 1. Key stakeholders groups represented in purposive interview sample

\begin{tabular}{lll}
\hline Leadership & \\
\hline Administration and & - & Department chairs \\
faculty governance & - & Dean's education innovation council \\
& - Faculty council committees: academic standing, \\
& - admissions, curriculum and evaluation \\
Faculty & - MS and GME education directors \\
Medical students & - Pociety of teaching scholars \\
& - Praditional curriculum \\
\hline
\end{tabular}

\section{Data analysis}

Two rounds of interviews were coded using a memoing strategy. ${ }^{29}$ Round 1 consisted of Nov-Dec interviews (3 months following the introduction of the NIC), and Round 2 included April-June interviews. Coding memos were pre- 
printed to parallel the AI interview protocol questions, the coder's initials, and space to record the randomly assigned protocol number and key findings (one memo per finding). Twenty-three Round 1 interviews (12 students and 11 faculty) were successfully recorded, transcribed, and subsequently coded (1 faculty digital recording malfunctioned). After initial Round 1 coding, authors worked in dyads to cluster memos using an affinity approach and then identify cluster themes using constant comparative methodology. All authors collaborated to develop a final list of key Round 1 features associated with educational program success. The process was repeated for Round 2 coding, building on and revising Round 1 themes as needed. Seventeen interviews ( 8 faculty, 5 EC students, 4 NIC students) were coded and analyzed in Round 2. Further interviews were not performed as saturation was reached.

\section{Results}

Analysis revealed five thematic clusters from Round 1, which were re-affirmed in Round 2, along with a sixth theme focused on the promotion of professional identity. Each theme is described with illustrative quotes from student and/or faculty success stories. As the themes were universal across EC and NIC participants and consistent with our IRB exemption to assure the identity of participants is protected, only limited participant identifiers (e.g., student, faculty, and role) are reported with the quote.

\section{Shared responsibility for learning}

All participants' stories exemplified an educational environment in which participants were actively engaged and collegial with shared responsibility for learning. Responsibility for learning was described when students learned with other students in and out of the classroom (e.g., studentcreated learning groups), as faculty met and worked with other faculty (e.g., workgroups, committee meetings), and/or student/faculty engaged together in shared learning. "When things are going well" per a course/clerkship leader, "both students and faculty are engaged. We have the opportunity to have both the basic scientist and the clinician in the room at the same time... (We) didn't just provide the answers, a discussion ensued, students are talking exchanging thoughts and generating new ideas. The faculty lead the discussions, provide their expertise, and listen to the students...this was one of the hardest things I had to learn...” was letting students be responsible. Students too feel "responsible for their learning". Faculty are no longer the "one way source" of information, as one student described. As students "we are actively involved, taking time to look things up." It's all about the "process of learning".

\section{Lived sense of community and collaboration}

Both students and faculty identified a pervasive sense of connectedness to the medical education program as well as to each other. Descriptions highlighting this sense of community went beyond the sense of shared responsibility and ownership in teaching and learning processes highlighted in theme 1 to emphasis the concept of learning with another in a collegial atmosphere. Students learned with, not just from, faculty, and faculty learned with other faculty, specifically when basic science and clinical faculty worked together. Students described positive, rather than competitive interactions with fellow students both inside and outside the classroom. Fellow students were no longer trying to "one up" their peers. Students reported that they are more comfortable taking risks, not afraid to say things or ask questions in front of their peers, not afraid to make mistakes, and are not worried about impressing their peers. The opportunity of having small groups allowed the students to "build trust with each other and the faculty". As one student emphasized, "I need a community of people around me and I need to be attuned to them and their strengths and weaknesses in order to best serve the population at large".

Faculty, too, valued the sense of community and collaborative learning between students. One faculty member described the use of senior students to teach junior students: "The excellent students also teach the junior students and take them under their wing and teach them some skills." In sum, successes highlighted in this theme emphasized actions were associated with a "lived" engagement of students and teachers in a collaborative community.

\section{Trust and respect in relationships}

Collegial and collaborative environments, per success stories, emerged from trusting and respectful relationships. At the foundation of these relationships was participants' sense of trust, which then "expanded" to the school's community as a whole. Numerous successes emphasized the personal, 1-on-1 contacts including teacher/student and/or more intense mentor/mentee connection. One student declared that these relationships are "a big part of early student-faculty interaction." Another student stated, "When students and faculty are engaged in mutual and respectful curiosities, the faculty are learning from the students...getting new ideas. It is a proven thing that naïve people can see things in the world that experienced people don't because their lenses are different. That it's a dynamic, reciprocal, respectful relationship with people having a good time and working hard".

\section{Active learning environments}

Within the confines of this discussion, the term active learning can be assumed to include all environments in which learners participate in meaningful learning activity requiring them to think about the activities in which they are participating. One student explained the importance of active learning in a safe learning environment: "It's good because...you learn by trial and error, and if you just learn from the books, you can only get so much." The most encouraging learning encounters involved active participa- 
tion from all involved in the setting, creating safe and meaningful environment for inquiry and innovation.

Having the opportunity for early clinical encounters was repeatedly the focus of student success stories. Beyond its active learning emphasis supporting the application of basic science knowledge, clinical experiences also fostered camaraderie, networking and professional development, increasing the overall sense of connectedness. This contextual learning further enhanced student and faculty engagement in the curriculum.

Numerous adjectives were used to describe features of active learning environments including "enthusiastic" and "engaged" learners and teachers. A faculty member described her high point moment as "seeing... the majority of the students are enthusiastic and engaged," while a student stated "you need to have an engaged faculty" to activate learning. Students recognize this active engagement. "I think when the teachers and the students are both enjoying what they are doing... you can tell, I mean we've had some teachers that just, they're really enjoying teaching us and we really enjoy being taught be them, like there's really good reciprocity going on..."

\section{Faculty commitment to student learning}

Students' and education directors' stories often identified faculty members who exhibited a level of excitement and sustained commitment to teaching and its continuous improvement as a strategy to maximize student learning. "...You can tell when that's happening in the classroom setting", per one student, because "I feel like I'm learning the most from the situation and the teachers are giving a great teaching experience. I know they're doing it because they love to teach. I think when that's going on I feel like the education is at its peak". These faculty members were described as "truly caring about student learning", "clearly communicating their expectations on a daily basis to their learners", "adapting their teaching to learner level", and taking "risks" to support student learning (e.g., allowing learner to go in and see a patient on their own, trying a new teaching approach). Teachers' clear commitment to student learning yielded results and was a repeated high point amongst teachers: "Seeing the lights go on and the excitement...seeing the excitement of the students as they understand the material..."

\section{Professional identity formation - physicians and teachers}

During Round 2 interviews, students and faculty success stories often included approaches to managing stresses that increase as the year progressed, recognizing that managing stress is essential in the medical profession. Positive interactions with faculty and patients reaffirmed students' identity as future medical professionals, mitigating some of the challenges and anxieties associated with medical education. Keeping an "eye on the prize" mentality was described as contributing to a positive outlook, particularly during stressful periods. Another student noted, "I think that the take home message for me as a critical life lesson of becoming a doctor is that I cannot go at this by myself... that as a physician I don't try to learn everything but I figure out how I can access the people who do know the information I do need best... You can't help other people if you're only helping yourself. That's kind of a message of medical school too. We are all studying seemingly for ourselves and then after four years we start to get to give back to the community, which is really wonderful. But at the same time I think it's really good that I've learned this lesson of I can't do this by myself. I need a community of people around me and I need to be attuned to them and their strengths and weaknesses in order to best serve the population at large."

Faculty members also described successes associated with their identities as educators and learners, emphasizing the importance of a long-term perspective as it "doesn't always go perfectly the first time" you try something new.

\section{Three tenets}

Common to each of these themes are three cross-cutting tenets associated with medical student education program success independent of curriculum model or student/faculty role: community, collaboration, and communication amongst and between students and faculty. These central tenets will be further elaborated upon in the discussion section.

\section{Discussion}

Change succeeds when the initiative is aligned with participants' beliefs and feelings. ${ }^{12}$ Student and faculty success stories reveal the behaviors, actions and results that they believe are associated with medical student education program success. Our thematic analysis of these stories identified six themes associated with program success: (1) Shared responsibility for learning; (2) lived community and collaboration; (3) trust and respect in relationships; (4) active learning environments; (5) faculty commitment to student learning; and (6) Professional identity formation: physicians and teachers.

Our themes map to three cross-cutting tenets associated with medical student education program success (community, collaboration, and communication amongst and between students and faculty), consistent with the recent Carnegie Foundation Educating Physicians report that calls for reforms in medical education. Cooke, Irby, and O'Brien emphasize the need for teachers and learners to be actively engaged in a collaborative learning community which supports standardization of outcomes and individualization of learning, integration of formal knowledge and clinical experience, habits of inquiry and innovation, and a focus on professional identify formation. ${ }^{5}$ It is reassuring to confirm that student and faculty descriptions of medical education programs depicting us "at our best" are consistent with 
evidence-based recommendations for medical education, thus adding validity to our findings.

Previous studies have associated burnout and depression to the lack of social interaction and limited student/faculty longitudinal relationships. ${ }^{30}$ The stress which occurs during periods of curriculum change may, as our success stories highlight, be attenuated by highlighting the opportunities to build and sustain faculty/student relationships through education workgroups and curriculum committees, as well as education through the use of interactive teaching strategies. ${ }^{31}$ More specifically, our results on engagement, relationships, active learning, and identify formation for teachers and future physicians reveal that the learning environment extends beyond the formal curriculum. The impacts of informal, unplanned, in-the-hall, cafeteria, spur-of-the moment encounters that connect medical students to faculty can, overtime, develop into rich mentoring relationships. Throughout our success descriptions, relationships between students and faculty were noted as key factors for both personal and professional growth and, as evidence indicates, contribute to successful careers in medicine..$^{32,33}$

These data are of particular interest for burgeoning medical education programs, as well as those currently implementing curricular changes. Both faculty and student expectations have evolved to a more interactive learning environment that focuses on intrapersonal interactions and building trust-based relationships. These expectations directly dictate specific elements of curricula that should be ubiquitous with the methodology utilized in classroom and clinical learning scenarios matched to the themes and tenets of community, collaboration, and communication.

Findings from this study must be considered in light of limitations commonly associated with qualitative studies including sample size, setting, and potential interviewer/analysis bias. To address these limitations, our broadbased workgroup used a purposive sampling approach drawing from our large volunteer pool to assure that a breadth of perspectives were represented in the narratives (e.g., EC, NIC, students, faculty, varying specialties, years of experience as teaching, leadership roles). Our study involved participants at one medical school and we while sought to minimize bias by having multiple interviewers and dyads to cluster memos, the findings should be interpreted acknowledging these limitations. However the consistency of our themes and tenets with recent work by Cooke et al. ${ }^{5}$ supports the generalizability of our findings.

\section{Conclusions}

As during any change initiative, curriculum change can bring fear and anxiety due to the lack of structure and control over one's environment and expectations for new roles. ${ }^{12}$ Through appreciative inquiry interviews, we were able to identify crosscutting positive themes associated with success in two medical education programs. Explicitly recognizing and highlighting these shared success tenets (community, collaboration, communication) and their underlying themes (e.g., engagement, professional identity, faculty/student relationships, active learning, excitement and willingness to teach) when faculty and students are engaged in designing and implementing "new curriculum" can promote a greater sense of shared purpose ${ }^{34}$ and ease stress associated with curriculum change.

\section{Acknowledgments}

The authors wish to thank Michael Olivier $\mathrm{PhD}$, Andrea Winthrop MD, Patricia Lye MD, and Kurt Pfeifer MD for continuous service as interviewers, and Sara Riordan and Julie-Ann Varona, transcriptionists.

This project was partially supported by the Elsa B. and Roger D. Cohen MDs Children's Hospital of Wisconsin/Medical College of Wisconsin Endowed Summer Fellowship in Medical Education which provided partial support for the first author as a medical student to participate in this project and the Educational Leadership for the Health of the Public-Research and Education Initiative Fund, a component of the Advancing a Healthier Wisconsin endowment at the Medical College of Wisconsin.

\section{Conflict of Interest}

The authors declare that they have no conflict of interest.

\section{References}

1. Dezee K, Artino AR, Elnicki DM, Hemmer PA, Durining SJ. Medical education in the United States of America. Medical Teacher. 2012;34:521525.

2. Beck, AH. The Flexner report and the standardization of American medical education. Journal of the American Medical Association. 2004;291(17):2139-2140.

3. Skochelak SE. A decade of reports calling for change in medical education. Academic Medicine. 2010;85(9):S26-S33.

4. Lueddeke GR. Transforming medical education for the 21st century. London: Radcliffe Publishing; 2012.

5. Cooke M, Irby D, O'Brien B. Educating physicians: a call for reform of medical school and residency. Carnegie Foundation for the Advancement of Teaching. San Francisco: Jossey-Bass; 2010.

6. Dennick R. Twelve tips for incorporating educational theory into teaching practices. Medical Teacher. 2012;33:618-624.

7. Irby DM, Cooke M, O'Brien BC. Calls for reform of medical education by the Carnegie Foundation for the advancement of teaching: 1910 and 2010. Academic Medicine. 2010;85(2):220-227.

8. Miller BM, Moore DE, Stead WW, Balser JR. Beyond Flexner: a new model for continuous learning in the health professions. Academic Medicine. 2010; 85(2): 266-272.

9. Schermerhorn JR, Hunt JG, Osborn RN. Organizational behavior. New York: John Wiley \& Sons, Inc; 2005.

10. Kotter JP. Leading change: why transformation efforts fail. Harvard Business Review. 1995;73(2):59-67.

11. Waterman RH, Peters TJ, Phillips JR. Structure is not organization. Business Horizons. 1980;23(3):14-26.

12. Duck JD. Managing change: The art of balancing. Harvard Business Review. 1993;71(6): 109-118.

13. Smollan RK, Sayers JG. Organizational culture, organizational change and emotions: a qualitative study. Journal of Change Management. 2009;9(4):435-457.

14. Banadaranayake, Raja C. The integrated medical curriculum. London: Radcliffe Publishing;2011. 
15. Broyles, India, Savidge M, Schwalenberg-Leip E, Thompson K, Lee R, Sprafka S. Stages of concern during curriculum change. Journal of the International Association of Medical Science Educators. 2007;17(1):14-26.

16. Cottingham AH, Suchman AL, Litzelman DK, Frankel RM, Mossbarger DL, Williamson PR, et al. Enhancing the informal curriculum of a medical school: a case study in organizational culture change. Journal of General Internal Medicine. 2008;23(6):715-22.

17. Whitney D, Trosten-Bloom A, Rader K. Appreciative leadership: focus on what works to drive winning performance and build a thriving organization. New York: McGraw-Hill; 2010.

18. Cooperrider DL, Srivastva S. Appreciative inquiry in organizational life. Research in Organizational Change and Development. 1987;1:129-269.

19. Whitney D, Trosten-Bloom A. The power of appreciative inquiry: a practical guide to positive change. San Francisco: Berrett-Koehler Publishers, Inc; 2010.

20. Quaintance JL, Arnold L, Thompson GS. What students learn about professionalism from faculty stories: an "appreciative inquiry" approach. Academic Medicine. 2010;85(1):118-23.

21. Richer M, Richie J, Marchionni C. If we can't do more, let's do it differently! Using AI to promote innovative ideas for better health care work environments. Journal of Nursing Management. 2009;17(8):947-955.

22. Helms AS, Perez TE, Baltz J, Donowitz G, Hoke G, Bass EJ, et al. Use of an appreciative inquiry approach to improve resident sign-out in an era of multiple shift changes. Journal of General Internal Medicine. 2011; 27(3):287-291.

23. Dematteo D, Reeves S. A critical examination of the role of appreciative inquiry within an interprofessional education initiative. Journal of Interprofessional Care. 2011;25(3):203-208.

24. Suchman AL, Williamson PR, Litzelman DK, Frankel RM, Mossbarger DL, Inui TS, et al. Toward an informal curriculum that teaches professionalism: transforming the social environment of a medical school. Journal of General Internal Medicine. 2004;19:501-504.
25. Litzelman DK, Cottingham AH. The new formal competency-based curriculum and informal curriculum at Indiana University School of Medicine: overview and five-year analysis. Academic Medicine. 2007; 82(4):410-21.

26. Cottingham AH, Suchman AL, Litzelman DK, Frankel RM, Mossbarger DL, Williamson PR, et al. Enhancing the informal curriculum of a medical school: a case study in organizational culture change. Journal of General Internal Medicine. 2008; 23(6):715-722.

27. Bell MA, Griffin WJ, Greene JY, Brokaw JJ. Medical students' reactions to a competency-based curriculum: one school's experience. Journal of the Internal Association of Medical Science Educators. 2008;18(1):21-27.

28. Mason M. Sample size and saturation in $\mathrm{PhD}$ studies using qualitative interviews. Forum: Qualitative Social Research. 2010;11(3).

29. Bower DJ, Young S, Larson G, Simpson D, Tipnis S, Begaz T, et al. Characteristics of patient encounters that challenge medical students' provision of patient-centered care. Academic Medicine. 2009; 84(10Suppl) :S74-S78.

30. Prince M. Does active learning work? A review of the research. Journal of Engineering Education. 2004; 93(3):223-231.

31. Bicket M, Misia S, Wright S, Shochet R. Medical student engagement and leadership within a new learning community. BMC Medical Education. 2010;10(20).

32. Haidet P, Stein HF. The role of the student-teacher relationship in the formation of physicians: The hidden curriculum as a process. Journal of General Internal Medicine. 2006;21(S1):S16-S20.

33. Hafler J, Ownby A, Thompson B, Fasser C, Grigsby K, Haidet P. Decoding the learning environment of medical education: a hidden curriculum perspective for faculty development. Academic Medicine. 2011;86(4):440-444.

34. E. Todres M, Tsimtsiou Z, Sidhu K, Stephenson A, Jones R. Medical students' perceptions of the factors influencing their academic performance: An exploratory interview study with high-achieving and re-sitting medical students. Medical Teacher. 2012;34: e325-e331. 